\title{
Pulverizing Characteristic of Woody Biomass by a Kind of Impact Mill
}

\author{
Chengbao $\mathrm{Wu}^{1}$, Guosheng $\mathrm{Gai}^{2}$, Yufeng Yang ${ }^{2}$, Zhixin Shang ${ }^{2}$
}

1. School of Aircraft Maintenance Engineering, Guangzhou Civil Aviation College, Guangzhou, 510430, China

\section{School of Materials Science and Technology, Tsinghua University, Beijing 10084, China}

Keywords: Wood biomass, Pulverizing characteristic, Impact mill, Pulverizing mechanism

\begin{abstract}
To investigate the pulverizing characteristic of wood biomass, the dried woody chip and the wood chip with four different moisture content of $5,10,15,20 \mathrm{wt} \%$ were chose as the raw materials, a kind of impact mill, the universal mill, was used to pulverize the wood biomass at different feed rates, the particle sizes (characteristic particle size and average particle size), uniformity coefficient, density, aspect ratio of wood powder and the specific energy consumption were measured, the pulverizing mechanism was also discussed. The results indicate that the specific energy consumption is in a range from 100 to $400 \mathrm{kWh} / \mathrm{t}$, and decreases with the increasing feed rate without changing of the basic motor power; the characteristic particle size is about $625 \mu \mathrm{m}$, the average particle size is $470 \mu \mathrm{m}$ or so, and both characteristic particle size and average particle size increase with the increasing feed rate, and then decrease before the feed rate reaches to the maximum capacity of the mill; the uniformity coefficient is range from 1.30 to 1.50 ; the bulk density is about $0.20 \mathrm{~g} / \mathrm{cm}^{3}$, the tap density is about $0.30 \mathrm{~g} / \mathrm{cm}^{3}$, both bulk density and tap density increase with the increase of the feed rate; the aspect ratio is in a range from 2.45 to 2.90 randomly with a common tendency of declining slightly following the increase of feed rate; the pulverizing mechanism of the biomass is the cross section of breakage.
\end{abstract}

\section{Introduction}

The biomass energy was investigated intensively and widely for the use of biomass energy does not increase carbon dioxide emissions and does not contribute to the risk of global climate change. In addition, using biomass to produce energy is often a way to dispose of waste materials that otherwise would create environmental risks [1]. It was predicted that $40 \%$ of the total global energy consumption would be from biomass energy in 2050 [2, 3].

Before being supplied to product energy, the biomass materials were pulverized. J. Kobayashi studied the drying process of woody biomass for fine grinding and the drying characteristics [4]. He found that grinding properties of woody biomass depend largely on its moisture content; the drying behavior of woody biomass and the effect of moisture content were also analyzed. He also carried out the simultaneous drying and pulverization of woody biomass using a continuous vibration mill. Luo [5] investigated the pulverizing process of different of biomass and compared the differences of the chemical constituents in two kinds of impacting mills. The result indicated that there were great differences in the energy required by the different biomasses for their unlike physical and mechanical properties, and the pulverization of woody biomasses such as pine chips requires more energy due to its higher lignin content. A. L. Bridgeman made an experimental investigation of the pulverization behavior of two energy crops, namely: willow and Miscanthus [6], he found that the torrefied biomass can be successfully pulverized and studied the torrefication process again and show the same results to save the pulverizing energy [7].

As mentioned above, the moisture content is one of important effects in the pulverizing characteristic of biomass. The objectives of this work are to investigate the pulverizing characteristic of wood biomass at different feed rate of a kind of impact mill and moisture content conditions.

\section{Experiments}




\section{Raw materials}

The biomass used for the experiment is woody chip from the furniture making factory, and its particle size were about $5 \mathrm{~mm}$.

After picked out the sand, metal scrap and wood blocks, the woody chip was separated evenly on the plastic sheet and dried naturally for one day in the sunshine. Then, the woody chip was put into the plastic bags for storage.

\section{Sample preparation}

To investigate the moisture content of the woody chip on the pulverizing characteristics, the dried woody biomass was spayed certain amount of water for pulverizing experiment. The moisture contents of the materials were $5,10,15$, and $20 \mathrm{wt} \%$ respectively.

After kept in plastic bags for 24h to make the moisture homogeneously, the water-bearing woody biomass were prepared to be pulverized.

\section{Instruments and methodology}

The universal mill, one kind of impact mill, is used as pulverizing machine in this work; the main motor power of the mill is $3 \mathrm{~kW}$, the diameter of the size control sieve, which is mounted inside the mill, is $1 \mathrm{~mm}$. The processing chart of the universal mill as shown in Fig.1:

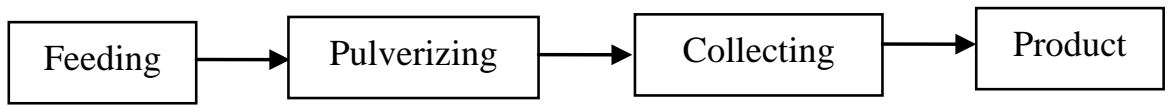

Fig.1. the pulverizing process chart for the universal mill

\section{Sample Analysis}

Sampling. Dividing the product of the experiments by the following method until in to $1 \mathrm{~kg}$ sample, Put the biomass product on a piece of plastic sheet to form a cone, then use a board to press it as a circular truncated cone. Cut the circular truncated cone into four equal portions across centre shaft, and then remove the two diagonal parts. Heap the remaining two parts into a new cone, and cut into four parts again by the above method. Stop the sampling until the remaining biomass product was about 300g.

Particle size distribution. The particle size distribution is analyzed by screen method with the sieves of Chinese civil engineering standard, similar to ASTM standard. The Tap Shaker Machine supplied by Wuxi Jianyi Experiment Instrument Co., Ltd. (ZBSX-92A, China) was used to sieve the sample, and get the mass portion of the particles with different diameter.

Density. Density is an important concept regarding storage transportation and packaging. In this study, both tap density and apparent density of the wood powder were measured by Powder Integrative Characteristic Tester supplied by Dandong Bettersize Science and Technology Co., Ltd. (BT-1000, China).

Aspect ratio. The aspect ratio of wood powder is the ratio of its longer dimension to its shorter dimension. It may be applied to two characteristic dimensions of the wood powder's three-dimensional shape. The Wireless Digital Microscope (2.4GHZ, Japan) and Image Process Plus (IPP 4.5.1, US) were used to measure the aspect ratio of the wood powder product.

Moisture content. the moisture content of the woody product was measured by the Moisture Meter(SFY20A, China )

\section{Results and discussion}

\section{The specific energy consumption}

It is one of the important cost elements. When evaluating a new product, the specific energy consumption is always one of prime questions that have to be taken consideration by the company. The specific energy consumption of the woody powder prepared by the universal mill was shown in Fig.2. 


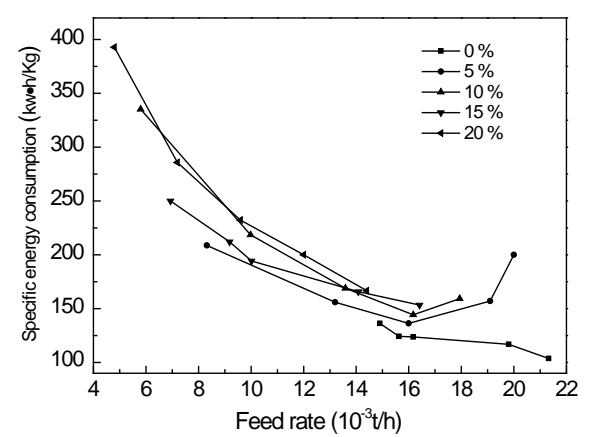

Fig.2. the relationship between the feed rate and the specific energy consumption

It can be seen from Fig.2 that the specific energy consumption is varied with the feed rate (capacity) and the moisture content. The specific energy consumption is getting less and less with the increase of capacity; it is the common sense to increase the processing capacity of the mill without changing of the basic motor power. Moisture has an important influence on the energy consumption through the decrease of capacity. It is obviously reason of the material grind ability decreased. With the moisture content of $20 \%$, the mill even cannot work above the minimum capacity of the material in dry state. With the moisture content of $5-10 \%$, the specific energy consumption will be increased when the capacity is getting close to the maximum capacity, the material is accumulated inside the mill, and much more energy is used in the friction not the breakage process of the biomass particles. The temperature inside the mill and the electricity current of the motor are getting higher and higher.

In general, moisture content is the key parameter which has a heavy influence on the mill performance, as the biomass particle is getting tenacity with the water makes the cellulose and lignin swollen inside the bio-cell.

\section{Particle size}

The particle size including characteristic particle size and average particle size is one of important parameters for evaluating the properties of the powder, is also one of important technological parameters for optimizing the product processing. In order to understanding the influence of operating condition to the product size distribution, we used the RRB (Rosin Rammler Bennet) size distribution modeling method to analyze the grinding process, with the same feed, check the product size distribution, under different grinding condition, as the Eq. 1 shows:

$$
x(D)=100 \exp \left[-\left(\frac{D}{D_{e}}\right)^{n}\right]
$$

Where the $D$ is the particle size, $\mu \mathrm{m} ; x(D)$ is the weight percentage which is above the particle size $D$ in the product, \%; $D_{e}$ is the characteristic particle size as the $x(D)$ is $36.8 \%, n$ is the uniformity coefficient. $D_{e}$ and $n$ reflect the influence of material properties and the features of the grinding machine.Fig.6 (a) showed the characteristic particle size of biomass product. After substituting the $D_{e}$ and the $n$ to the Eq.1, one can get the average particle size of the biomass powder, as Fig.3 shows:

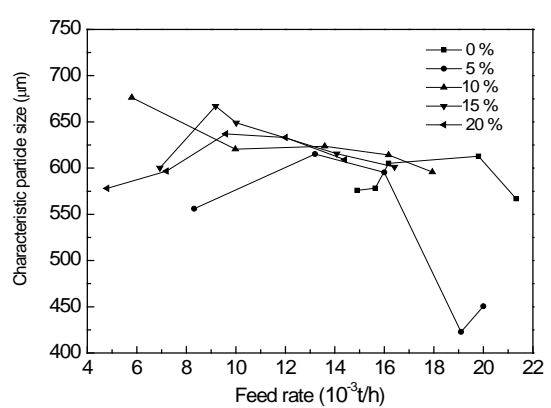

(a)

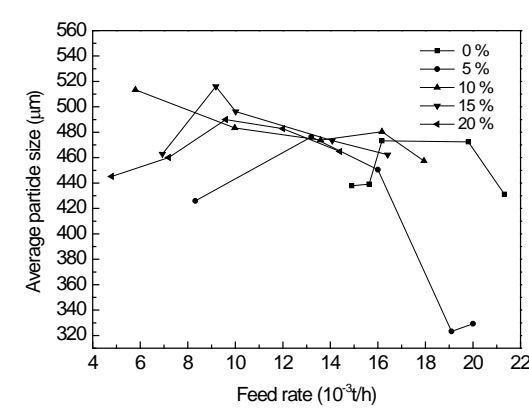

(b)

Fig.3. the effect of the feed rate on the characteristic particle size and the average particle size. (a) the characteristic particle size,(b) the average particle size 
From Fig.3, it can be seen that the particle size is getting larger and larger along with the increasing feed rate, and then getting less and less before the feed rate reaches to the maximum capacity of the mill, the average characteristic particle size is about $625 \mu \mathrm{m}$, the average particle size is about $470 \mu \mathrm{m}$. There are two kinds of grinding process inside the mill, one is the impact of the hammer to the biomass particle and the other is the friction (or shear) between the biomass particles and the hammers. If the feed rate is lower, the material remained in the mill is less; the impact force is the dominant grinding action inside the mill. As the feed rate increased, the material residence is getting higher and higher, the friction or shear action will become dominant in the mill.

For the entire test with different moisture materials, the rule is the same: as the feed rate increasing, the input energy is dispersed to the increased resident material; the particle size is getting coarser. When the material accumulated inside the mill the friction force can make the particle to be finer and finer. With the decreasing grind ability of biomass, the particle size is getting larger and larger, following the increase of moisture content.

Since the particle size is finally controlled by the aperture diameter of the sieve (screen) inside the mill, the product size is not changed greatly.

\section{Uniformity coefficient}

The uniformity coefficient $n(-)$ in Eq. 1 reflects the size wideness of particle distribution in the expression of Eq. 1. The higher it is, the narrower the size distribution. The uniformity coefficient of the biomass powder was shown in Fig.4.

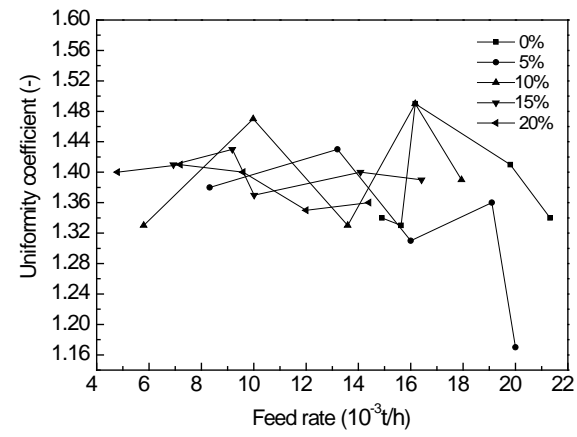

Fig.4 The effect of the feed rate on the uniformity coefficient of the woody powder

The uniformity coefficient is in a range from 1.30 to 1.50 , and is seemly no regulation in the curves in Fig.4. The reason maybe the product particle size is controlled by the aperture of the inside sieve; and when the feed rate increased, the uniformity coefficient is getting smaller,.

\section{Density}

The bulk density and tap density of the biomass powder were shown in Fig.5, respectively.
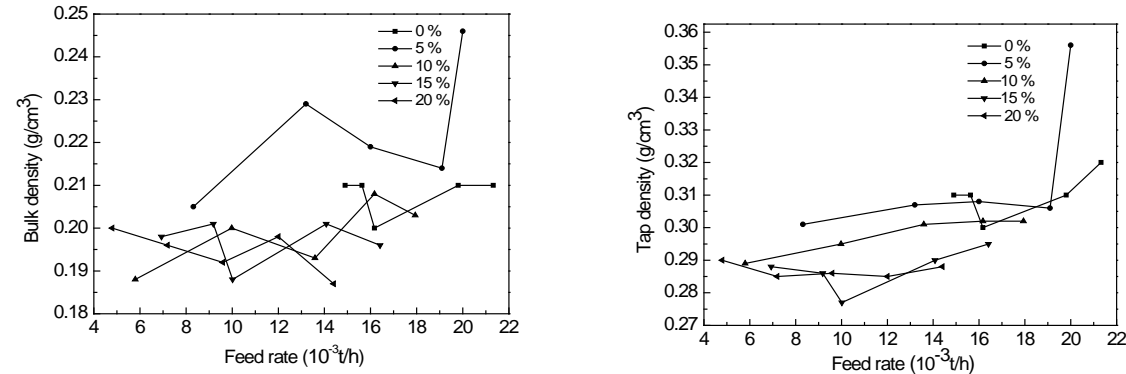

Fig.5. the effect of the feed rate on the bulk density and the tap density. (a) The bulk density, (b) the tap density

Form Fig.5, we can know that the main tendency of the densities is getting bigger and bigger along with the feed rate. The bulk density is getting less and less along with the moisture content under the same feed rate. It is the reason that as the increase of moisture, the material is become swollen and the volume getting larger and larger. The tap density has a clear relation with the moisture content, especially; some random error is revised during the tapping process. Compared the densities of materials with the moisture content of 0 and 5\%, the relation between moisture content and bulk density is change at this point. These mean if there is some amount of water inside 
the biomass, the size distribution of the product will changed, and the bulk density can be increased in some degree.

\section{Aspect ratio}

It is one of parameters for quantitatively characterizing the morphology of the powder, which reflects the basic property of the material and the pulverizing mechanism.

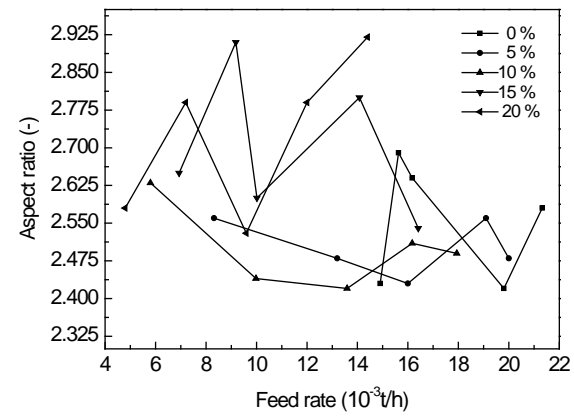

Fig.6. the relationship between the feed rate and aspect ratio of the biomass powder

From Fig.6, It can be found that the aspect ratio is in a range from 2.45 to 2.90 randomly with a common tendency of declining slightly following the increase of feed rate. It may be the reason that when the feed rate is higher, more material is accumulated inside the mill, the friction (shear) action is stronger, this action often cause the biomass particle to break along the grain direction. To study the aspect ratio with pulverizing parameter, is for the purpose of understanding how the pulverizing energy is used to break the particle in the middle vertically to the grain direction or to break the particle in along the grain direction.

\section{Conclusions}

Moisture content of the biomass material has an important influence on the specific energy consumption through the decrease of capacity, and the specific energy consumption is getting less and less with the increasing capacity of the mill. The particle size is getting larger and larger along with the increasing feed rate, and then getting less and less before the feed rate reaches to the maximum capacity of the mill. When the feed rate increased, the uniformity coefficient is getting smaller. The main tendency of the densities is getting bigger and bigger along with the feed rate. The bulk density is getting less and less along with the moisture content under the same feed rate. The aspect ratio declines slightly with the increasing feed rate. The pulverizing mechanism of the biomass is the cross section of breakage.

\section{References}

[1] G. Weber, Q. Fu \& H. Wu, Energy efficiency of an integrated process based on gasification for hydrogen production from biomass, Asia-Pacific Journal of Chemical Engineering, 14(1-2) , pp33-48,2006.

[2] Zhang Xiliang, Chen Rong \& He Jiankun, the Role of public policy in commercializing gasification-based biomass power generation technology in China, Acta Energiae Solaris Sinica, 25(4),pp557-560.

[3] Liu Baoliang, Jiang Jianchun, Research and Development of biomass gasification technique for power generation in China, Biomass Chemical Engineering, 40(4), pp47-52, 2006.

[4] J. Kobayashi, Y. Itaya, S. Tsukada, K. Mizuno, M. Ueda, H. Morikawa, T. Sugimoto, Y. Ueda, Y. Oshika, N. Kobayashi, S. Hatano \& S. Mori, Drying technology for woody biomass for fine grinding by vibration mills, Asia-Pacific Journal of Chemical Engineering, 2, pp83-89, 2007.

[5] Siyi Luo, Chang Liu, Bo Xiao \& Lei Xiao, A novel biomass pulverization technology, Renewable Energy, 36(2),pp578-582, 2011.

[6] T.G. Bridgeman, J.M. Jones, A. Williams \& D.J. Waldron, An investigation of the grindability of two torrefied energy crops, Fuel, 89(12),pp 3911-3918,2010. 
[7] Vincent Repellin, Alexandre Govin, Matthieu Rolland \& René Guyonnet, Energy requirement for fine grinding of torrefied wood, Biomass \& Bioenergy, 34, pp923-930, 2010. 